\title{
Light inactivation of metronidazole sensitivity discs
}

\author{
P. H. JONES AND ANNE P. SCOTT \\ From the Public Health Laboratory, Lewsey Road, Luton, LU4 ODZ, UK
}

SUMMARY We found that daylight inactivated metronidazole sensitivity discs. They should therefore be stored in light-proof containers.

Increasing awareness of the importance of anaerobic infections has led to the widespread use of in-vitro sensitivity testing of anaerobes against metronidazole. Moreover, the use of metronidazole discs as an aid in the presumptive recognition of anaerobes on primary isolation plates is a standard procedure in some laboratories.

We and other laboratories have noticed that some metronidazole discs are inactive, so that anaerobic organisms appear to be resistant and anaerobes on primary isolation appear to be facultatively anaerobic. This has led to a widespread appreciation of disc-failure, and we know that at least one laboratory routinely uses multiple metronidazole discs to obviate this problem.

Since metronidazole is heat-stable it has been suggested that the sensitivity discs may be inactivated by light, although at the time of writing no formal study has been carried out. We report the results of an investigation of the effect of various types of light on metronidazole discs, with special reference to inactivation of the drug.

\section{Material and methods}

We used Metronidazole Sensitivity Discs $5 \mu \mathrm{g}$ (Mast Laboratories), lot numbers 28827 and 32068, expiry dates 1 April 1978 and 1 December 1978, that had been stored at $4^{\circ} \mathrm{C}$ in the dark.

Sensitivity plates were prepared from Oxoid Sensitest Agar (Batch No. 248 17539) with 5\% defibrinated horse blood added and dispensed in $20-\mathrm{ml}$ volumes in $9 \mathrm{~cm}$ petri dishes.

The organism used for sensitivity testing was Bacteroides fragilis is fragilis (NCTC 9343).

The following light sources were employed: tungsten filament lamp $60 \mathrm{~W}$, fluorescent strip lamp $30 \mathrm{~W}$, ultraviolet lamp $15 \mathrm{~W}$ (Philips TUV $15 \mathrm{~W}$ ) of

Received for publication 26 April 1977 emission wavelength $253.7 \mathrm{~nm}$ (unused), and daylight.

Discs were cut in half and each half placed in a $\vec{\circ}$ separate Mast disc container from which the label $\infty$ had been removed. Up to 13 half discs were held in $ᄋ$ each container. The two halves of a single disc (test and control) were identifiable by an inert coloured $z$ spot code. Test halves were then exposed to the following types of light at the distances stated: tungsten lamp, $22 \mathrm{~cm}$; fluorescent lamp, $80 \mathrm{~cm}$; $\mathbb{\Phi}$ ultraviolet lamp, $30 \mathrm{~cm}$. In addition one set of half discs was exposed to natural light $15 \mathrm{~cm}$ from a cleas glass window. The containers were shaken once day to redistribute the half discs. The exposus times to each type of light were recorded.

In all experiments the containers of control half discs were placed in light-proof cardboard boxes near the tests. At the end of the light exposure periods each test half disc was matched with its unexposed control half and the activity of both was measured by conventional disc sensitivity testing against the indicator strain of $\boldsymbol{B}$. fragilis, as outlined below. Additional control discs that had not been exposed to light were cut in half and the activity of each half measured by the same disc diffusion technique. This set consisted of equal numbers of discs from the manufacturer's two lots.

Two series of experiments were performed. In the first, which occupied a 14-day period, separate sets of 25 half discs were exposed intermittently for 96 hours to a single artificial light source or for $113 \fallingdotseq$ hours to daylight. Test and control half discs were $\tilde{N}$ held at ambient temperature during the whole of this period. In the second series of experiments sets of 25 half discs were exposed for varying periods $c$ (up to 96 hours) to daylight during a 10-day period. In both of these experiments test half discs were $O$ placed in light-proof boxes at the end of their $\mathbb{D}$ exposure times. All half discs (tests and controls) were then examined together for activity on completion of the experiment. 
The activity of the half discs was measured by a standard disc diffusion technique (Garrod et al., 1973), freshly prepared sensitivity plates being flooded with an appropriate suspension of $B$. fragilis. Since $5-\mu \mathrm{g}$ discs of metronidazole commonly produce very wide zones of inhibition (up to $50 \mathrm{~mm}$ ) each sensitivity plate conveniently accommodated only two half discs-a test half disc and its corresponding control. Plates were incubated at $37^{\circ} \mathrm{C}$ for 18 hours in an anaerobic atmosphere containing $10 \%$ of carbon dioxide. Callipers were used to measure the zone diameters of inhibition.

\section{Results}

The mean diameter of the zones of inhibition produced by the 100 control half discs tested after no exposure to light was $34.7 \mathrm{~mm}$ (range $39.7 \mathrm{~mm}$ $27.9 \mathrm{~mm}$ ). The two halves of one exceptional disc produced zones of only $13.7 \mathrm{~mm}$ and $15.3 \mathrm{~mm}$. The mean difference in the zone diameters obtained from the two halves of the 50 discs was $1.3 \mathrm{~mm}$ (range $0.1 \mathrm{~mm}-3.7 \mathrm{~mm}$ ).

In the first experiment all the control half discs produced zones of inhibition comparable to those of the general controls noted above. All of the 25 test halves that had been exposed to daylight, however, produced zones that were smaller than their corresponding controls. Five of them produced no zones and the reduction in zone size was more than $10 \mathrm{~mm}$ in eight others. In contrast, all the test halves that had been exposed to artificial light sources showed little or no change in activity.

The results of the second experiment, summarised in the Table, show that with an increasing duration of exposure to daylight there was a corresponding decrease in the activity of the test halves compared with their controls. After 96 hours' exposure, for example, 21 of 25 half discs had no detectable activity against the indicator organism. Clearly, however, this loss of activity was not an 'all-or-none'

Table Effect of exposure to daylight on the actiuity of metronidazole half discs

Exposure to No. of half discs showing a reduction in diameter of

daylight zones of inhibition of $(\mathrm{mm})$ :

$\begin{array}{lllllll}\text { (h) } & \leqslant 4.9 & 5-9.9 & 10-14.9 & 15-19.9 & 20-25 & \text { Nilzone }\end{array}$

\begin{tabular}{lrrrrrr}
\hline $24^{*}$ & 11 & 12 & - & - & - & - \\
$48 \dagger$ & 2 & 6 & 9 & 6 & - & 1 \\
72 & 1 & 4 & 7 & 3 & 4 & 6 \\
96 & - & - & - & 2 & 2 & 21 \\
\hline
\end{tabular}

*Two test half discs were bigger than controls by $0.7 \mathrm{~mm}$ and $1.9 \mathrm{~mm}$ respectively.

+One test half disc was bigger than control by $0.9 \mathrm{~mm}$. phenomenon since discs showed variation in the size of the zone of inhibition. These results suggest that inactivation probably began during the first 24-hour period of exposure, since the mean reduction in zone size $(4.5 \mathrm{~mm})$ was much greater than the mean difference in zone size of unexposed general controls $(1.3 \mathrm{~mm})$.

\section{Discussion}

We intended to investigate the possibility of light inactivation of metronidazole discs under conditions relevant to those in most routine situations. The types of light chosen for exposure studies were those commonly used in a microbiology laboratory, although the distances were convenient but arbitrary. The discs were biologically tested since this mimicked normal laboratory practice.

Exposure to daylight resulted in a variable but often total loss of observable activity of the half discs tested and it was not surprising to find that during the second experiment, in which many discs became totally inactive, there was more direct sunlight on the discs. No attempt was made to identify the responsible fraction of light. That some of the discs were not inactivated may have been due to uneven exposure in the disc containers, though the latter were shaken at regular intervals. Although long exposure to daylight was necessary for the complete inactivation of most of the half discs in a set reduction in activity began after only 24 hours' exposure. One of the discs tested produced a much reduced zone even without exposure, suggesting either inactivation before receipt or incomplete dosing of the disc with the metronidazole during manufacture.

The findings of this investigation re-emphasise the need for proper control of antimicrobial sensitivity testing - as is provided, for example, by Stokes's method, in which each disc is 'internally' controlled. In this way the use of multiple metronidazole discs as referred to earlier is obviated. We recommend that metronidazole discs should be kept in a light-proof container.

We thank Dr A. T. Willis for help and guidance and Mrs J. Holt for secretarial assistance.

\section{Reference}

Garrod, L. P., Lambert, H. P., and O'Grady, F. (1973). Antibiotic and Chemotherapy, 4th edn, pp. 493-494. Churchill Livingstone, Edinburgh. 